OPEN ACCESS

Edited by:

Carlos Tomaz,

Universidade Ceuma, Brazil

Reviewed by:

Abbas Haghparast,

Shahid Beheshti University of Medical

Sciences, Iran

Dennis Qing Wang,

Zhujiang Hospital of Southern Medical

University, China

*Correspondence:

Dongliang Jiao

jd13925697@163.com

${ }^{\dagger}$ These authors have contributed equally to this work

Received: 25 August 2018 Accepted: 05 December 2018 Published: 18 December 2018

Citation:

Zhai C, Cui M, Cheng X, Ao X, Zhao T, Wu W, Shao $Q, G e D$, Song $H, Q i F$, Ling Q, Ma M, Xu M and Jiao D (2018) Vitamin B12 Levels in

Methamphetamine Addicts. Front. Behav. Neurosci. 12:320 doi: 10.3389/fnbeh.2018.00320

\section{Vitamin B12 Levels in Methamphetamine Addicts}

\author{
Changping Zhai ${ }^{1+}$, Ming Cui ${ }^{1+}$, Xiaodong Cheng ${ }^{2+}$, Xiang Ao ${ }^{1+}$, Tingting Zhao ${ }^{1}$, Wei Wu ${ }^{1}$, \\ Qun Shao ${ }^{1}$, Dexue $\mathrm{Ge}^{1}$, Hongmei Song ${ }^{1}$, Fangzhi $\mathrm{Qi}^{2}$, Qiang Ling ${ }^{2}$, Mengdi Ma ${ }^{3}$, \\ Mengyuan $\mathrm{Xu}^{3}$ and Dongliang Jiao ${ }^{3 *}$ \\ ${ }^{1}$ Anhui Province Veterans Hospital, Bengbu, China, ${ }^{2}$ Compulsory Isolated Drug Rehabilitation Center, Bengbu, China, \\ ${ }^{3}$ Department of Psychiatry, Bengbu Medical College, Bengbu, China
}

Objective: It has been established that reduced vitamin B12 serum levels are associated with cognitive decline and mental illness. The chronic use of methamphetamine (MA), which is a highly addictive drug, can induce cognitive impairment and psychopathological symptoms. There are few studies addressing the association of MA with vitamin B12 serum levels. This study examined whether the serum levels of B12 are associated with MA addiction.

Methods: Serum vitamin B12, homocysteine (Hcy), glucose and triglyceride concentrations were measured in $123 \mathrm{MA}$ addicts and 108 controls. In addition, data were collected on their age, marital status, level of education and Body Mass Index (BMI) for all participants. In the patient group, the data for each subject were collected using the Fagerstrom Test for Nicotine Dependence (FTND), the Alcohol Use Disorders Identification Test (AUDIT), and a drug use history, which included the age of onset, total duration of MA use, the number of relapses and addiction severity.

Results: Our results showed that MA addicts had lower vitamin B12 levels $(p<0.05)$ than those of healthy controls, but Hcy levels were not significantly different between the two groups $(p>0.05)$. Serum B12 levels were negatively correlated with the number of relapses in the MA group. Furthermore, binary logistics regression analysis indicated that the B12 was an influencing factor contributing to addiction severity.

Conclusion: The findings of this study suggest that some MA addicts might have vitamin B12 deficiency, and serum B12 levels may be involved in the prognosis of MA addiction.

Keywords: methamphetamine, addiction, neurotoxicity, vitamin B12, homocysteine

\section{INTRODUCTION}

The problem of addiction to amphetamine-type stimulants (ATS) has become a global public health problem. The most common ATS is methamphetamine (MA). MA is highly addictive and can lead to psychiatric illnesses or cognitive defects(Curran et al., 2004; Scott et al., 2007; Jacobs et al., 2008). Psychotic symptoms induced by ATS include hallucinations, delusions (Bramness et al., 2012), depression (Sutcliffe et al., 2009), and anxiety(Bagheri et al., 2015). A strong relationship between cognitive defects or psychotic symptoms and substance abuse relapse or prognosis has been demonstrated in laboratory and clinical studies; treatment of cognitive and mental disorders may contribute to the prognosis of addiction (Liu et al., 2016). However, the mechanism of 
cognitive dysfunction and psychiatric illnesses induced by MA use has not been fully explained, and there has been a lack of effective medicines to treat ATS-related problems.

It has been proposed that a decreased B12 level is responsible for neuropsychiatric disorders (Kumar, 2014). For example, B12 levels were lower (Kim et al., 2008; Saedisomeolia et al., 2011; Almeida et al., 2015) in patients with schizophrenia and affective disorders compared to those in healthy individuals. Reduced B12 levels may affect the brain through multiple mechanisms and are involved in the occurrence of disease (Tangney et al., 2011). There is a great deal of evidence suggesting that vitamin B12 supplementation may be important for treating certain patients (Moore et al., 2012; Brown and Roffman, 2014). However, there have never been any reports examining whether serum levels of B12 are associated with ATS addiction.

The causes of B12 deficiency are multifactorial, including nutritional causes due to poor dietary intake, as well as functional causes due to impairments in absorption and intracellular processing and trafficking. Vitamin B12 is a vitamin that requires an intestinal secretion (endogenous factor) to be absorbed. Therefore, conditions associated with malabsorption, such as ileal disease or resection, may cause B12 deficiency (Carmel, 2000). MA can induce intestinal disease (Beyer et al., 1991; Herr and Caravati, 1991; Brannan et al., 2004; Prendergast et al., 2014). Thus, chronic long-term abuse of MA may cause nutrient absorption disorders. Recently, it has been reported that B12 may alleviate MA-induced neurotoxicity (Moshiri et al., 2018). Therefore, we speculate that MA abuse may affect the absorption of B12 and lead to B12 deficiency.

Homocysteine (Hcy) is a thiol group-containing amino acid that naturally occurs in all humans. An elevated level of Hcy can often be caused by deficiencies in vitamin B12. Elevated Hcy could induce oxidative stress (Papatheodorou and Weiss, 2007), lipid metabolism imbalance (Liao et al., 2006), N-methyld-aspartate receptor overexcitation (Kim and Pae, 1996), tau hyperphosphorylation and accumulation (Wei et al., 2011) and upregulation of hepcidin (Luo et al., 2016), which may lead to cognitive deficits, such as Alzheimer's disease (Xu et al., 2012; Castillo-Carranza et al., 2017; Sternberg et al., 2017; Wang et al., 2017) and psychiatric disorders (Osher et al., 2008; Kinoshita et al., 2013). Homocysteine has been a potential predictor for dementia (Zou et al., 2018). Thus, we hypothesized that B12 and Hcy may be involved in MA addiction-related problems.

The aim of the present study was to examine (1) whether serum levels of B12 and Hcy in MA-dependent patients are different from healthy controls and (2) whether there is any relationship between MA addiction severity and the levels of B12 and Hcy.

\section{METHODS}

\section{Participants}

A total of 123 male MA addicts was recruited from the Compulsory Isolated Drug Rehabilitation Center in Bengbu. The MA addicts who participated in this study met the diagnosis of MA dependence according to the Diagnostic and Statistical Manual of Mental Disorders (DSM-V) criteria. The diagnoses were verified by a senior psychiatrist with the rank of associate professor.

The control group (108 men) was recruited from staff members of the Bengbu Mental Health Center and the local community, and none of them had any history of drug use. The controls were matched with the MA groups in terms of gender, age and education. All subjects in the study participated voluntarily and signed an informed consent form for the protocol. The study was approved by the Institutional Review Board (permission number: 2017-53) of Bengbu Medical University. All experiments were carried out in accordance with the approved guidelines and regulations.

Blood samples were collected on the first day when the participants came into the center, and the relevant indicators were tested on the same day. The inclusion criteria were (1) no history of severe mental illness, (2) age between 18 and 45 years old, (3) normal vision and hearing, (4) more than 9 years of education, and (5) abstinent from MA for $<3$ months.

The exclusion criteria were (1) psychiatric or neurological illness (e.g., schizophrenia, affective disorders, stroke, seizure, or parkinsonism); (2) other chronic diseases (e.g., diabetes, hypertension, hyperlipemia, and gastroenteropathy); (3) other substance dependence (e.g., opioids and cocaine), except for cigarettes and alcohol, within the past 5 years; and (4) being a vegetarian.

\section{Clinical and Neuropsychological Assessment}

Before the tests, detailed questionnaires, including a complete medical history, physical and psychological examination, were obtained from each subject.

All participants filled out a self-administered case report form including their age, marital status, and level of education. In the patient group, each subject was interviewed by one psychiatrist, and data were collected on the Fagerstrom Test for Nicotine Dependence (FTND), the Alcohol Use Disorders Identification Test (AUDIT), and a drug use history, which included the age of onset, total duration of MA use, the number of relapses, etc. Addiction severity was assessed using a self-administered case report form that included questions about subjective craving feelings and the degree of drug dependence. Simultaneous Body Mass Index (BMI = weight/height squared) was measured in the field.

\section{Assessment of Serum B12, Hcy, Glucose, and Triglyceride Levels}

Fasting blood samples were collected from each participant between 7:00 and 9:00 a.m. (before breakfast) for the measurement of 4 markers, including B12, Hcy, glucose and triglyceride. Serum concentrations of these markers were measured $3 \mathrm{~h}$ later by a technician who was blinded to the diagnostic status of the subjects. Hcy, glucose and triglyceride levels were measured using a HITACHI 7600 automatic biochemistry analyzer (Hitachi High-Technologies Corporation, Japan), and the B12 levels were measured using a MAGLUMI 2000 automatic chemiluminescence immunoassay analyzer 
TABLE 1 | Demographics and drug use history data, quartile $(25,50,75)$ or Mean \pm SD (standard deviation).

\begin{tabular}{|c|c|c|c|c|}
\hline Variable & MA group $(n=123)$ & HC group $(n=108)$ & $F / Z / x^{2}$ & $p$ \\
\hline Age (years) (mean \pm SD) & $34.20 \pm 7.99$ & $35.75 \pm 12.28$ & 22.40 & 0.27 \\
\hline Years of education (years) $(25,50,75)$ & $1.00,2.00,2.00$ & $2.00,5.00,6.00$ & -10.18 & 0.00 \\
\hline Married (\%) & $96(78.0 \%)$ & $80(74.0 \%)$ & 0.50 & 0.54 \\
\hline BMl (mean \pm SD) & $24.07 \pm 3.96$ & $24.02 \pm 2.54$ & 0.09 & 0.93 \\
\hline Glucose $(25,50,75)$ & $4.56,4.99,5.88$ & $4.91,5.12,5,50$ & -1.42 & 0.16 \\
\hline Triglyceride $(25,50,75)$ & $0.80,1.16,1.73$ & $0.96,1.35,2.07$ & -1.76 & 0.08 \\
\hline FTND score $(25,50,75)$ & $1.00,3.00,6.00$ & & & \\
\hline AUDIT score $(25,50,75)$ & $0.00,3.00,13.50$ & & & \\
\hline Age of onset (years) (mean \pm SD) & $26.24 \pm 7.61$ & & & \\
\hline The numbers of relapse $(25,50,75)$ & $1.00,1.00,2.00$ & & & \\
\hline Total duration of MA use (month) $(25,50,75)$ & $3.00,12.00,36.00$ & & & \\
\hline Addiction severity $(25,50,75)$ & $0.00,3.00,6.00$ & & & \\
\hline
\end{tabular}

FTND, Fagerstrom Test for Nicotine Dependence; AUDIT, The Alcohol Use Disorders Identification Test; BMI, Body Mass Index.

(New Industries Biomedical Engineering Co., Ltd., Shenzhen, China).

\section{Statistical Analysis}

The data were analyzed using SPSS, version 16.0 (SPSS Inc., an IBM Company Headquarters, 233 S. Wacker Drive, 11th floor Chicago, Illinois 60606). Group differences were compared using the Student's $t$-test for continuous variables, the nonparametric Mann-Whitney $U$-test for abnormal distribution variables and the chi-squared test for categorical variables. The correlation between variables was studied using Spearman's correlation. A binary logistics regression analysis was done with addiction severity as the dependent factor and age, education, age of onset, total duration of MA use, number of relapses, AUDIT score, FTND score, B12, and Hcy as independent factors. The alpha level was reported with $p<0.05$ (two-sided tests) considered to be statistically significant.

\section{RESULTS}

\section{Demographics and Drug Use History Data (Table 1)}

There were no differences in age, marital status, BMI, glucose or triglyceride between the two groups $(p>0.05)$. The MA group had fewer years of education. Information on smoking and alcohol consumption in the controls was unavailable. The MA group results had either quartiles or a mean for FTND score $(1.00,3.00,6.00)$, AUDIT score $(0.00,3.00,13.50)$, age of onset (years) (26.24 \pm 7.61$)$, the number of relapses $(1.00,1.00$, $2.00)$, total duration of MA use (months) $(3.00,12.00,36.00)$, and addiction severity $(0.00,3.00,6.00)$.

\section{Vitamin B12 and Hcy Levels in MA and Healthy Controls (Table 2)}

The quartiles for vitamin B12 levels were (224.70, 328.00, 424.70) $\mathrm{pg} / \mathrm{mL}$ in the MA group and $(341.4,390.1,542.80) \mathrm{pg} / \mathrm{mL}$ in the control group. Vitamin B12 levels were significantly lower in the MA group than in healthy controls $(Z=-5.37, p=0.000)$. Hcy levels were not significantly different between the two groups ( $p$ $>0.05$ ).

\section{The Association Between B12 and Hcy Levels With Drug Use History, FTND Score and AUDIT Score in the MA Group}

A significant, negative correlation was observed between B12 and the number of relapses $(r=-0.308, \mathrm{df}=121, p=0.001)$ (Figure 1) in the MA group. There was no significant correlation between Hcy levels and drug use history in the MA group (all $p>$ 0.05). There was no significant correlation between B12 and Hcy levels with the FTND or AUDIT scores in the MA group. These results suggest that the more relapses an individual had, the more significant the impact was on the decline of B12.

\section{Influencing Factors on Addiction Severity}

FTND, AUDIT, age of onset, the number of relapses, total duration of MA use, B12 and Hcy were taken as independent variables, and the addiction severity was taken as a dependent variable for binary logistic regression analysis. The obtained logistic model had statistical significance, $x_{(7)}^{2}=17.176$, $P=0.016$. B12 (beta $\left.=-0.006, \operatorname{Exp}_{(\mathrm{B})}=0.994, p=0.038\right)$ and FTND (beta $=0.220, \operatorname{Exp}_{(\mathrm{B})}=1.246, p=0.036$ ) were statistically significant among the seven independent variables (or predictors) included in the model.

\section{DISCUSSION}

The major findings of this study were that (1) B12 levels were significantly reduced in MA-dependent patients compared the healthy controls, (2) the decrease in B12 was correlated with the number of relapses, and (3) the decrease in B12 was a factor influencing the severity of drug addiction.

To the best of our knowledge, this study was the first to find that serum B12 levels were significantly lower in MAdependent patients compared to those in healthy controls. B12 is absorbed from the intestine, and it is a vitamin that requires an intestinal secretion (intrinsic factor) to be absorbed. It is 
TABLE 2 | Plasma vitamin B12, Hcy levels in MA and healthy controls.

\begin{tabular}{lcccc}
\hline & $\begin{array}{c}\text { MA group }(\boldsymbol{n}=\mathbf{1 2 3}) \\
(\mathbf{2 5}, \mathbf{5 0}, \mathbf{7 5})\end{array}$ & $\begin{array}{c}\text { HC group }(\boldsymbol{n}=\mathbf{1 0 8}) \\
(\mathbf{2 5}, \mathbf{5 0 , 7 5 )}\end{array}$ & $\boldsymbol{z}$ & $\boldsymbol{p}$ \\
\hline $\begin{array}{l}\mathrm{B} 12 \\
(\mathrm{pg} / \mathrm{mL})\end{array}$ & $224.70,328.00,424.70$ & $341.4,390.1,542.80$ & -5.37 & 0.00 \\
$\begin{array}{l}\mathrm{Hcy} \\
(\mu \mathrm{moi} / \mathrm{L})\end{array}$ & $10.80,14.20,24.30$ & $12.50,14.20,17.40$ & -0.33 & 0.74 \\
\hline
\end{tabular}

affected by intestinal function (Lima et al., 2018); therefore, intestinal diseases may lead to B12 absorption disorders. MA could cause splanchnic vasoconstriction and necrotizing angiitis (Johnson and Berenson, 1991; Kelly et al., 1992), which can even induce intestinal ischemia and infarction (Beyer et al., 1991; Herr and Caravati, 1991; Brannan et al., 2004; Prendergast et al., 2014). Thus, chronic long-term abuse of MA could cause nutrient absorption disorders, weight loss and malnutrition. For example, previous studies found that the serum folic acid in MAdependent patients was significantly lower than in the controls (Nakazawa et al., 1981), and folic acid uptake was decreased by MA in primary cultures of human syncytiotrophoblasts (Keating et al., 2009). Although we did not test folic acid levels, we found that B12 levels were significantly decreased in MA-dependent patients; therefore, we speculated that the cause of decreased serum B12 levels might due to a disorder in B12 absorption caused by MA.

In addition, there was no significant difference in Hcy levels between the MA group and the control group in this study. Hcy is metabolized through two major pathways: transsulfuration and methylation. Approximately $50 \%$ is catabolized in the transsulfuration pathway, which does not require B12 (BolanderGouaille, 2002). The results suggest that Hcy may not be involved in the mental disorders and cognitive impairment induced by MA.

MA, as a nervous stimulant, can induce severe neurotoxicity by oxidative stress (Solhi et al., 2014; Zhang et al., 2018), damage to the blood-brain barrier (Northrop and Yamamoto, 2015) and overexpression of pro-inflammatory cytokines (Tocharus et al., 2010), which leads to subsequent psychiatric disorders or cognitive deficits (Curran et al., 2004). Vitamin B12 is essential for the development and initial myelination of the central nervous system, and vitamin B12 deficiency can lead to a wide range of neurological symptoms (Lövblad et al., 1997). A decrease in vitamin B12 may be involved in MAinduced neurotoxicity in the following ways. (1) Overexpression of pro-inflammatory cytokines. It was reported that a low B12 level was associated with overexpression of pro-inflammatory cytokines (Politis et al., 2010). Pro-inflammatory cytokines might be involved in the pathophysiology of many psychiatric conditions (Banerjee et al., 2017; Vakilian et al., 2018). (2) Mitochondrial DNA disorders. Vitamin B12 plays an important role in the maintenance of mitochondrial genome integrity (Fenech, 2012). B12 deficiency could induce mitochondrial DNA sequence variation and might contribute to cognitive function disorders and psychiatric symptoms (Inczedy-Farkas et al., 2012; Tranah et al., 2012; Zaia et al., 2017). (3) Promoting oxidative

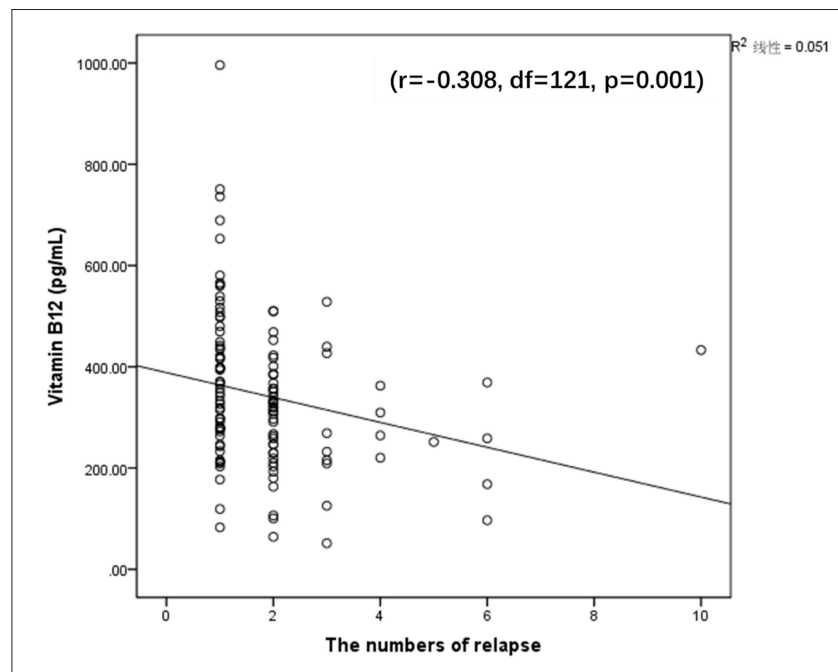

FIGURE 1 | Significantly negative correlation between vitamin B12 and the number of relapse $(r=-0.308, \mathrm{df}=121, p=0.001)$.

stress. Vitamin B12 deficiency could also induce neurotoxicity by promoting oxidative stress (Misra et al., 2016; Zhang et al., 2016; Bito et al., 2017). (4) Damaging the blood-brain barrier. Bloodbrain barrier pathology is recognized as a central factor in the development of many neurological disorders. Experimental and human evidence support the idea that vitamin B12 plays a role in maintaining the integrity of the blood-brain barrier (Lehmann et al., 2003; Pieters et al., 2009; Pollak et al., 2018). Neurotoxicity induced by $\mathrm{B} 12$ deficiency might contribute to various types of neuropsychiatric disorders (Bell et al., 1991; Türksoy et al., 2014; Zhang et al., 2016) and cognitive impairment (Wang et al., 2001; Tangney et al., 2011). In conclusion, Vitamin B12 deficiency might be involved in MA-induced neurotoxicity in these ways and induce mental disorders as well as cognitive impairment.

However, there is little research on how b12 was involved in the formation of MA addiction. According to previous research, we speculate that cognitive impairment and psychiatric disorders, which reflect damage to brain regions associated with addiction (Everitt et al., 2007), could exacerbate drug addiction (Chambers et al., 2009) and disrupt treatment and prognosis (Aharonovich et al., 2006; Witkiewitz and Bowen, 2010; Hellem, 2016). In addiction, it has been reported that decreased vitamin B12 availability induces ER stress (Ghemrawi et al., 2013). Our previous study (Jiao et al., 2017) showed that ER stress was involved in the process of MA addiction. Thus, decreased vitamin B12 might be directly involved in the formation of MA addiction through ER stress.

The current study suggests that B12 was associated with the number of relapses and is one of the important factors influencing the severity of addiction. These results suggest that a decrease of B12 may affect the prognosis of addiction. Recent research has reported (Moshiri et al., 2018) that B12 at safe doses may be a promising treatment for MA-induced brain damage through inhibition of neuron apoptosis and increasing 
the reduced GSH level. B12 could serve as a novel therapeutic agent against MA-induced neurotoxicity.

The shortcomings of this study were that participants were not tested for their cognitive and psychosis status, which is a major concern among addicts. Thus, it is necessary to perform additional studies to explore whether the decreased vitamin B12 levels correlate with cognitive dysfunction and psychosis in MA addicts. Some reports show that the consumption of cigarettes and wine correlates with decreased vitamin B12 (Seethalakshmi and Chinnaswamy, 2006; Haj Mouhamed et al., 2011; Fragasso, 2013). However, the present study did not find that the consumption of cigarettes and wine were correlated with plasma vitamin B12 in MA addicts; therefore, it is necessary to compare MA addicts with the healthy group to confirm the associations between these factors.

\section{ETHICS STATEMENT}

All subjects in the study were voluntary and signed an informed consent for the protocol. The study was approved by the Institutional Review Board of Bengbu Medical University

\section{REFERENCES}

Aharonovich, E., Hasin, D. S., Brooks, A. C., Liu, X., Bisaga, A., and Nunes, E. V. (2006). Cognitive deficits predict low treatment retention in cocaine dependent patients. Drug Alcohol Depend. 81, 313-322. doi: 10.1016/j.drugalcdep.2005.08.003

Almeida, O. P., Ford, A. H., and Flicker, L. (2015). Systematic review and metaanalysis of randomized placebo-controlled trials of folate and vitamin B12 for depression. Int. Psychogeriatr. 27, 727-737. doi: 10.1017/S10416102150 00046

Bagheri, M., Mokri, A., Khosravi, A., and Kabir, K. (2015). Effect of abstinence on depression, anxiety, and quality of life in chronic methamphetamine users in a therapeutic community. Int. J. High Risk Behav. Addict. 4:e23903. doi: 10.5812/ijhrba.23903

Banerjee, A., Khemka, V. K., Roy, D., Dhar, A., Sinha Roy, T. K., Biswas, A., et al. (2017). Role of pro-inflammatory cytokines and vitamin D in probable Alzheimer's Disease with depression. Aging Dis. 8, 267-276. doi: 10.14336/AD.2016.1017

Bell, I. R., Edman, J. S., Morrow, F. D., Marby, D. W., Mirages, S., Perrone, G., et al. (1991). B complex vitamin patterns in geriatric and young adult inpatients with major depression. J. Am. Geriatr. Soc. 39, 252-257. doi: 10.1111/j.1532-5415.1991.tb01646.x

Beyer, K. L., Bickel, J. T., and Butt, J. H. (1991). Ischemic colitis associated with dextroamphetamine use. J. Clin. Gastroenterol. 13, 198-201. doi: 10.1097/00004836-199104000-00016

Bito, T., Misaki, T., Yabuta, Y., Ishikawa, T., Kawano, T., and Watanabe, F. (2017). Vitamin B12 deficiency results in severe oxidative stress, leading to memory retention impairment in Caenorhabditis elegans. Redox Biol. 11, 21-29. doi: 10.1016/j.redox.2016.10.013

Bolander-Gouaille, C. (2002). "The homocysteine metabolism," in Focus on Homocysteine and the Vitamins (Paris: Springer).

Bramness, J. G., Gundersen, O. H., Guterstam, J., Rognli, E. B., Konstenius, M., Loberg, E. M., et al. (2012). Amphetamine-induced psychosis-a separate diagnostic entity or primary psychosis triggered in the vulnerable? $B M C$ Psychiatry 12:221. doi: 10.1186/1471-244x-12-221

Brannan, T. A., Soundararajan, S., and Houghton, B. L. (2004). Methamphetamine-associated shock with intestinal infarction. Medgenmed 6:6. (permission number: 2017-53). All experimental procedures in this manuscript were in strict accordance with the approved guidelines and regulations.

\section{AUTHOR CONTRIBUTIONS}

$\mathrm{CZ}, \mathrm{MC}$, and DJ conceived and designed the experiments. CZ, TZ, WW, QS, DG, MM, and MX carried out experiments. $\mathrm{XA}, \mathrm{TZ}$, and HS analyzed experimental data. FQ, XC, and QL contributed reagents, materials, analysis tools. CZ and TZ wrote the first draft of the manuscript. DJ provided critical revision of the manuscript for important intellectual content.

\section{FUNDING}

Program of Bengbu Medical College Science and Technology Development (BYKF17113), Program of Bengbu Medical College Science and Technology (BYKY17186), Compulsory Isolated Drug Rehabilitation Center funded projects, Innovative training Program for Chinese College students (201810367006).

Brown, H. E., and Roffman, J. L. (2014). Vitamin Supplementation in the treatment of schizophrenia. Cns Drugs 28, 611-622. doi: 10.1007/s40263-014-0172-4

Carmel, R. (2000). Current concepts in cobalamin deficiency. Ann. Rev. Med. 51, 357-375. doi: 10.1146/annurev.med.51.1.357

Castillo-Carranza, D. L., Nilson, A. N., Van Skike, C. E., Jahrling, J. B., Patel, K., Garach, P., et al. (2017). Cerebral microvascular accumulation of tau oligomers in Alzheimer's Disease and related tauopathies. Aging Dis. 8, 257-266. doi: 10.14336/AD.2017.0112

Chambers, C. D., Garavan, H., and Bellgrove, M. A. (2009). Insights into the neural basis of response inhibition from cognitive and clinical neuroscience. Neurosci. Biobehav. Rev. 33:631. doi: 10.1016/j.neubiorev.2008.08.016

Curran, C., Byrappa, N., and Mcbride, A. (2004). Stimulant psychosis: systematic review. Br. J. Psychiatry 185, 196-204. doi: 10.1192/bjp.185.3.196

Everitt, B. J., Hutcheson, D. M., Ersche, K. D., Pelloux, Y., Dalley, J. W., and Robbins, T. W. (2007). The orbital prefrontal cortex and drug addiction in laboratory animals and humans. Ann. N. Y. Acad. Sci. 1121, 576-597. doi: 10.1196/annals.1401.022

Fenech, M. (2012). Folate (vitamin B9) and vitamin B12 and their function in the maintenance of nuclear and mitochondrial genome integrity. Mutat. Res. 733, 21-33. doi: 10.1016/j.mrfmmm.2011.11.003

Fragasso, A. (2013). "Vitamin B12 Deficiency in Alcoholics," in Alcohol, Nutrition, and Health Consequences. Nutrition and Health, eds R. Watson, V. Preedy and S. Zibadi (Totowa, NJ: Humana Press).

Ghemrawi, R., Pooya, S., Lorentz, S., Gauchotte, G., Arnold, C., Gueant, J., L., et al. (2013). Decreased vitamin B12 availability induces ER stress through impaired SIRT1-deacetylation of HSF1. Cell Death Dis. 4:e553. doi: $10.1038 /$ cddis. 2013.69

Haj Mouhamed, D., Ezzaher, A., Neffati, F., Douki, W., and Najjar, M. F. (2011). Effect of cigarette smoking on plasma homocysteine concentrations. Clin. Chem. Lab. Med. 49, 479-483. doi: 10.1515/cclm.2011.062

Hellem, T. L. (2016). A review of methamphetamine dependence and withdrawal treatment: a focus on anxiety outcomes. J. Subst. Abuse Treat. 71, 16-22. doi: 10.1016/j.jsat.2016.08.011

Herr, R. D., and Caravati, E. M. (1991). Acute transient ischemic colitis after oral methamphetamine ingestion. Am. J. Emerg. Med. 9, 406-409. doi: 10.1016/0735-6757(91)90073-S

Inczedy-Farkas, G., Remenyi, V., Gal, A., Varga, Z., Balla, P., Udvardymeszaros, A., et al. (2012). Psychiatric symptoms of patients with primary 
mitochondrial DNA disorders. Behav. Brain Funct. 8:9. doi: 10.1186/1744-90 81-8-9

Jacobs, E., Fujii, D., Schiffman, J., and Bello, I. (2008). An exploratory analysis of neurocognition in methamphetamine-induced psychotic disorder and paranoid schizophrenia. Cogn. Behav. Neurol. 21, 98-103. doi: 10.1097/WNN.0b013e31816bdf90

Jiao, D. L., Chen, Y., Liu, Y., Ju, Y. Y., Long, J. D., Du, J., et al. (2017). SYVN1, an ERAD E3 Ubiquitin ligase, is involved in GABAAalpha1 degradation associated with methamphetamine-induced conditioned place preference. Front. Mol. Neurosci. 10:313. doi: 10.3389/fnmol.2017.00313

Johnson, T. D., and Berenson, M. M. (1991). Methamphetamineinduced ischemic colitis. J. Clin. Gastroenterol. 13, 687-689. doi: 10.1097/00004836-199112000-00015

Keating, E., Gonçalves, P., Campos, I., Costa, F., and Martel, F. (2009). Folic acid uptake by the human syncytiotrophoblast: interference by pharmacotherapy, drugs of abuse and pathological conditions. Reproduct. Toxicol. 28, 511-520. doi: 10.1016/j.reprotox.2009.07.001

Kelly, M. A., Gorelick, P. B., and Mirza, D. (1992). The role of drugs in the etiology of stroke. Clin. Neuropharmacol. 15:249. doi: 10.1097/00002826-199208000-00001

Kim, J. M., Stewart, R., Kim, S. W., Yang, S. J., Shin, I. S., and Yoon, J. S. (2008). Predictive value of folate, vitamin B12 and homocysteine levels in late-life depression. Br. J. Psychiatry 192, 268-274. doi: 10.1002/(SICI)1097-0185(19990601)255:23.0.CO;2-L

Kim, W. K., and Pae, Y. S. (1996). Involvement of N-methyl-d-aspartate receptor and free radical in homocysteine-mediated toxicity on rat cerebellar granule cells in culture. Neurosci. Lett. 216, 117-120. doi: 10.1016/0304-3940(96)1 3011-1

Kinoshita, M., Numata, S., Tajima, A., Shimodera, S., Imoto, I., and Ohmori, T. (2013). Plasma total homocysteine is associated with DNA methylation in patients with schizophrenia. Epigenetics 8, 584-590. doi: 10.4161/epi.24621

Kumar, N. (2014). Neurologic aspects of cobalamin (B12) deficiency. Handbook Clin. Neurol. 120:915. doi: 10.1016/B978-0-7020-4087-0.00060-7

Lehmann, M., Regland, B., Blennow, K., and Gottfries, C. G. (2003). Vitamin B12-B6-folate treatment improves blood-brain barrier function in patients with hyperhomocysteinaemia and mild cognitive impairment. Dement. Geriatr. Cogn. Disor. 16, 145-150. doi: 10.1159/000071002

Liao, D., Tan, H., Hui, R., Li, Z., Jiang, X., Gaubatz, J., et al. (2006). Hyperhomocysteinemia decreases circulating high-density lipoprotein by inhibiting apolipoprotein A-I protein synthesis and enhancing HDL cholesterol clearance. Circ. Res. 99, 598-606. doi: 10.1161/01.RES.0000242559.42077.22

Lima, S., Webb, C. L., Deery, E., Robinson, C., and Zedler, J. A. Z. (2018). Human intrinsic factor expression for bioavailable vitamin B12 enrichment in microalgae. Biology 7:19. doi: 10.3390/biology7010019

Liu, Y., Shen, W. W., Huang, Y. Y., Zhang, J. B., and Zhou, W. H. (2016). Relapse motivation analysis for methamphetamine addicts. Chin. J. Clin. Pharmacol. 32, 1166-1168. doi: 10.13699/j.cnki.1001-6821.2016.13.004

Lövblad, K., Ramelli, G., Remonda, L., Nirkko, A. C., Ozdoba, C., and Schroth, G. (1997). Retardation of myelination due to dietary vitamin B12 deficiency: cranial MRI findings. Pediatr. Radiol. 27, 155-158. doi: 10.1007/s002470050090

Luo, X., Luo, Z., Zhang, Z., Yang, H., Lai, B., Yao, Q., et al. (2016). Homocysteine upregulates hepcidin expression through BMP6/SMAD signaling pathway in hepatocytes. Biochem. Biophys. Res. Commun. 471, 303-308. doi: 10.1016/j.bbrc.2016.02.001

Misra, U. K., Kalita, J., Singh, S. K., and Rahi, S. K. (2016). Oxidative Stress Markers in Vitamin B12 Deficiency. Mol. Neurobiol. 54, 1-7. doi: 10.1007/s12035-016-9736-2

Moore, E., Mander, A., Ames, D., Carne, R., Sanders, K., and Watters, D. (2012). Cognitive impairment and vitamin B12: a review. Int. Psychogeriatr. 24, 541-556. doi: 10.1017/s1041610211002511

Moshiri, M., Hosseiniyan, S. M., Moallem, S. A., Hadizadeh, F., Jafarian, A. H., Ghadiri, A., et al. (2018). The effects of vitamin B12 on the brain damages caused by methamphetamine in mice. Iran. J. Basic Med. Sci. 21, 434-438. doi: 10.22038/ijbms.2018.23362.5897

Nakazawa, Y., Yokoyama, T., Kurauchi, H., Ueda, S., Sakamoto, T., Imatoh, N., et al. (1981). Folic acid in serum and cerebrospinal fluid of chronic alcoholics and methamphetamine addicts. Drug Alcohol Depend. 7:193. doi: 10.1016/0376-8716(81)90033-8
Northrop, N. A., and Yamamoto, B. K. (2015). Methamphetamine effects on blood-brain barrier structure and function. Front. Neurosci. 9:69. doi: $10.3389 /$ fnins.2015.00069

Osher, Y., Bersudsky, Y., Silver, H., Sela, B. A., and Belmaker, R. H. (2008). Neuropsychological correlates of homocysteine levels in euthymic bipolar patients. J. Affect. Disor. 105, 229-233. doi: 10.1016/j.jad.2007.04.005

Papatheodorou, L., and Weiss, N. (2007). Vascular oxidant stress and inflammation in hyperhomocysteinemia. Antioxid. Redox Signal 9, 1941-1958. doi: 10.1089 /ars.2007.1750

Pieters, B., Staals, J., Knottnerus, I., Rouhl, R., Menheere, P., Kessels, A., et al. (2009). Periventricular white matter lucencies relate to low vitamin B12 levels in patients with small vessel stroke. Stroke 40, 1623-1626. doi: 10.1161/STROKEAHA.108.523431

Politis, A., Olgiati, P., Malitas, P., Albani, D., Signorini, A., Polito, L., et al. (2010). Vitamin B12 levels in Alzheimer's Disease: association with clinical features and cytokine production. J. Alzheimers Dis. 19, 481-488. doi: 10.3233/JAD-2010-1252

Pollak, T. A., Drndarski, S., Stone, J. M., David, A. S., Mcguire, P., and Abbott, N. J. (2018). The blood-brain barrier in psychosis. Lancet Psychiatry 5, 79-92. doi: 10.1016/S2215-0366(17)30293-6

Prendergast, C., Hassanein, A. H., Bansal, V., and Kobayashi, L. (2014). Shock with intestinal ischemia: a rare complication of methamphetamine use. Am. Surg. 80, 101-102.

Saedisomeolia, A., Djalali, M., Moghadam, A. M., Ramezankhani, O., and Najmi, L. (2011). Folate and vitamin B12 status in schizophrenic patients. J. Res. Med. Sci. Offic. J. Isfahan Univ. Med. Sci. 16(Suppl. 1), S437.

Scott, J. C., Woods, S. P., Matt, G. E., Meyer, R. A., Heaton, R. K., Atkinson, J. H. et al. (2007). Neurocognitive effects of methamphetamine: a critical review and meta-analysis. Neuropsychol. Rev. 17, 275-297. doi: 10.1007/s11065-007-9031-0

Seethalakshmi, S., and Chinnaswamy, P. (2006). Biochemical changes in alcoholism - vitamin B12 and folic acid levels in alcoholics. Ind. J. Nutr. Dietet. 43, 112-115. doi: 10.1097/00005176-200205000-00010

Solhi, H., Malekirad, A., Kazemifar, A. M., and Sharifi, F. (2014). Oxidative stress and lipid peroxidation in prolonged users of methamphetamine. Drug Metabol. Lett. 7, 79-82. doi: 10.2174/187231280702140520191324

Sternberg, Z., Hu, Z., Sternberg, D., Waseh, S., Quinn, J. F., Wild, K., et al. (2017). Serum hepcidin levels, iron dyshomeostasis and cognitive loss in Alzheimer's Disease. Aging Dis. 8, 215-227. doi: 10.14336/AD.2016.0811

Sutcliffe, C. G., German, D., Sirirojn, B., Latkin, C., Aramrattana, A., Sherman, S. G., et al. (2009). Patterns of methamphetamine use and symptoms of depression among young adults in northern Thailand. Drug Alcohol Depend. 101, 146-151. doi: 10.1016/j.drugalcdep.2008.11.014

Tangney, C. C., Aggarwal, N. T., Li, H., Wilson, R. S., Decarli, C., Evans, D. A., et al. (2011). Vitamin B12, cognition, and brain MRI measures: a cross-sectional examination. Neurology 77, 1276-1282. doi: 10.1212/WNL.0b013e3182315a33

Tocharus, J., Khonthun, C., Chongthammakun, S., and Govitrapong, P. (2010). Melatonin attenuates methamphetamine-induced overexpression of proinflammatory cytokines in microglial cell lines. J. Pineal Res. 48, 347-352. doi: 10.1111/j.1600-079x.2010.00761.x

Tranah, G. J., Nalls, M. A., Katzman, S. M., Yokoyama, J. S., Lam, E. T., Zhao, Y., et al. (2012). Mitochondrial DNA sequence variation associated with dementia and cognitive function in the elderly. J. Alzheimers Dis. 32, 357-372. doi: 10.3233/JAD-2012-120466

Türksoy, N., Bilici, R., Yalçiner, A., zdemir, Y., rnek, I., Tufan, A. E., et al. (2014). Vitamin B12, folate, and homocysteine levels in patients with obsessive-compulsive disorder. Neuropsychiatr. Dis. Treat. 2014, 1671-1675. doi: 10.2147/NDT.S67668

Vakilian, A., Razavi-Nasab, S. M., Ravari, A., Mirzaei, T., MoghadamAhmadi, A., Jalali, N., et al. (2018). Vitamin B12 in association with antipsychotic drugs can modulate the expression of Pro-/Anti-inflammatory cytokines in alzheimer disease patients. Neuroimmunomodulation 24, 310-319. doi: 10.1159/000486597

Wang, H. X., Wahlin, Basun, H., Fastbom, J., Winblad, B., and Fratiglioni, L. (2001). Low levels of vitamin B12 and folate and Alzheimer's disease incidence. Reply to Carlos R. M. Rieder, and Daniele Fricke. Neurology 57, 1742-1743.

Wang, R., Chen, Z., Fu, Y., Wei, X., Liao, J., Liu, X., et al. (2017). Plasma cystatin C and high-density lipoprotein are important biomarkers of alzheimer's disease 
and vascular dementia: a cross-sectional study. Front. Aging Neurosci. 9:26. doi: 10.3389/fnagi.2017.00026

Wei, W., Liu, Y. H., Zhang, C. E., Wang, Q., Wei, Z., Mousseau, D. D., et al. (2011). Folate/vitamin-B12 prevents chronic hyperhomocysteinemia-induced tau hyperphosphorylation and memory deficits in aged rats. J. Alzheimers Dis. 27, 639-650. doi: 10.3233/JAD-2011-110770

Witkiewitz, K., and Bowen, S. (2010). Depression, craving, and substance use following a randomized trial of mindfulness-based relapse prevention. J. Consul. Clin. Psychol. 78:362. doi: 10.1037/a0019172

Xu, Y., Yan, J., Zhou, P., Li, J., Gao, H., Xia, Y., et al. (2012). Neurotransmitter receptors and cognitive dysfunction in Alzheimer's disease and Parkinson's disease. Progr. Neurobiol. 97, 1-13. doi: 10.1016/j.pneurobio.2012.02.002

Zaia, A., Maponi, P., Di Stefano, G., and Casoli, T. (2017). Biocomplexity and fractality in the search of biomarkers of aging and pathology: Focus on Mitochondrial DNA and Alzheimer's Disease. Aging Dis. 8, 44-56. doi: 10.14336/AD.2016.0629

Zhang, K., Zhang, Q., Jiang, H., Du, J., Zhou, C., Yu, S., et al. (2018). Impact of aerobic exercise on cognitive impairment and oxidative stress markers in methamphetamine-dependent patients. Psychiatry Res. 266, 328-333. doi: $10.1016 /$ j.psychres.2018.03.032
Zhang, Y., Hodgson, N. W., Trivedi, M. S., Abdolmaleky, H. M., Fournier, M., Cuenod, M., et al. (2016). Decreased brain levels of vitamin B12 in aging, autism and schizophrenia. PLos ONE 11:e0146797. doi: 10.1371/journal.pone.0 146797

Zou, J., Chen, Z., Liang, C., Fu, Y., Wei, X., Lu, J., et al. (2018). Trefoil Factor 3, cholinesterase and homocysteine: potential predictors for parkinson's disease dementia and vascular parkinsonism dementia in advanced stage. Aging Dis. 9, 51-65. doi: 10.14336/AD.2017.0416

Conflict of Interest Statement: The authors declare that the research was conducted in the absence of any commercial or financial relationships that could be construed as a potential conflict of interest.

Copyright $\odot 2018$ Zhai, Cui, Cheng, Ao, Zhao, Wu, Shao, Ge, Song, Qi, Ling, Ma, $X u$ and Jiao. This is an open-access article distributed under the terms of the Creative Commons Attribution License (CC BY). The use, distribution or reproduction in other forums is permitted, provided the original author(s) and the copyright owner(s) are credited and that the original publication in this journal is cited, in accordance with accepted academic practice. No use, distribution or reproduction is permitted which does not comply with these terms. 